Original Article

\title{
Kinematic analysis of dance-based exergaming: effect of song pace on center of mass and joint mobility
}

\author{
Ernest Kwesi Ofori, PT, MS ${ }^{1}$, Savitha Subramaniam, PT, $\mathrm{MS}^{1)}$, Shuaijie Wang, $\mathrm{PhD}^{1)}$, \\ TAnvi Bhatt, $\mathrm{PhD}^{1)^{*}}$ \\ 1) Department of Physical Therapy, University of Illinois at Chicago: 1919 W Taylor St., Chicago, \\ Illinois 60612, USA
}

\begin{abstract}
Purpose] To establish a quantitative kinematic assessment method for examining postural stability and physical mobility during dance-based exergaming. [Participants and Methods] Fifteen young adults participated in one session of dance-based exergaming assessment using segments from Kinect 'Just Dance 3' consisting of slow-, medium-, and fast-paced songs. A motion capture system was used to record full body kinematics, and a customized MATLAB code was used to compute the variables of interest, such as center of mass sway area, excursion, and peaks, as well as step count and joint excursions. [Results] Total center of mass sway area and excursion were significantly greater for slow-paced (total sway area $=1,077.6 \pm 209.9 \mathrm{~cm}^{2}$; total excursion=629.8 $\pm 380.5 \mathrm{~cm}$ ) and fast-paced (total sway area $=314.1 \pm 133.6 \mathrm{~cm}^{2}$; total excursion $=478.5 \pm 149.0 \mathrm{~cm}$ ) songs than for the medium-paced song (total sway area $=212.9 \pm 346.0 \mathrm{~cm}^{2}$; total excursion $=311.2 \pm 119.5 \mathrm{~cm}$ ). Joint excursion was greater for the knee (ranging from: (slow to fast-paced songs: $55.5^{\circ}-83.1^{\circ}$ ) and hip (slow to fast-paced: $40.6^{\circ}-50.4^{\circ}$ ) than for the ankle (slow to fast-paced: $32.2^{\circ}-46.7^{\circ}$ ) for all three dance paces. Additionally, step count was significantly, positively correlated with song pace $(\mathrm{r}=0.52)$. [Conclusion] The current study was able to quantify and provide normative values for postural control and joint mobility among healthy young adults during dance-based exergaming with 3 hip-hop songs of different paces from the Microsoft Kinect 'Just Dance 3'. The results indicated that different paces (intensities) of dance songs corresponded to distinct movement kinematic trends, particularly with slow-paced song having the maximum center of mass excursion and lowest joint excursion, while fast-paced song exhibits the reverse, along with medium-paced song exhibiting the highest hip and ankle joint angle excursions, while the fast-paced song had increased knee joint angle excursions.

Key words: Dance, Exergaming, Postural control
\end{abstract}

(This article was submitted Dec. 8, 2018, and was accepted May 15, 2019)

\section{INTRODUCTION}

Alternative therapies, such as dance, have been shown to employ full-body movement practice to provide comprehensive rehabilitation by addressing balance, gait, strength, and physical performance in healthy and neurologically impaired populations $^{1-5}$. These studies have also been seen to facilitate positive exercise-related behaviors, such as motivation and compliance, towards interventions using dance therapy ${ }^{6,7)}$. Dance involves coordinated control of multiple limb segments, synchronized with a rhythmic stimulus, over many degrees of movement ${ }^{8)}$. When dancing, individuals undergo self-generated, repeated internal perturbations. Such self-induced perturbations require individuals to rapidly shift their center of mass $(\mathrm{CoM})$ to different spatial locations while preventing loss of balance. The aforesaid practice of rapidly shifting their CoM

*Corresponding author. Tanvi Bhatt (E-mail: tbhatt6@uic.edu)

(C2019 The Society of Physical Therapy Science. Published by IPEC Inc.

(c) (i) $\odot$ This is an open-access article distributed under the terms of the Creative Commons Attribution Non-Commercial No Deriva-

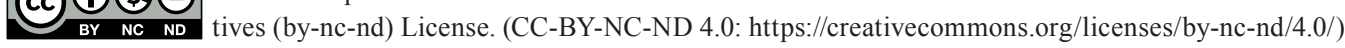


facilitates improvements in balance control and reduces the risk of falls $\left.{ }^{9}, 10\right)$. Recent studies have demonstrated that dance therapy can be successful for improving mobility deficits and balance control in various populations including healthy young adults, aging individuals, and people with disabilities ${ }^{11-14)}$. A systematic review evaluated the influence of dance on healthy older adults and found clinically meaningful benefits for fall-risk reduction factors such as balance, gait, strength, and physical function ${ }^{15}$. Additional systematic review and meta-analysis investigating the effectiveness of dance as an intervention for people with neurological deficits found improvements for clinical measures of balance control and quality of life $\mathrm{e}^{9,16,17)}$.

However, it is important to note that there is considerable diversity with regards to the types of dance styles which were used in previous studies, including Korean, Ballroom, Argentine, Tango, Turkish folkloristic, Greek, Caribbean, and Aerobic dance $^{8,9,18)}$. Such dance forms consist of diverse movement patterns (in anteroposterior (AP) and mediolateral (ML) directions) and paces, which alter the biomechanical and physiological demands, resulting in specific adaptations for each dance form ${ }^{19,20)}$. Yet, there is limited evidence of how the quality of movement and posture control differ for these various dance forms, as well as limited evidence as to how the rhythm and pace of dance affect the distinct domains of balance control. Therefore, it is difficult to quantify and relate clinical improvements to enhancements in actual movement control which may be critical for rehabilitation in aging individuals and those with neurological disorders. Additionally, although dance therapy delivered in the form of community-based group classes for a healthy living, prevention, and wellness, and/or rehabilitation has several benefits, there are some barriers associated with dance training such as the cost of, access to, transportation to, and caregiver support of therapy. Some of these limitations of structured or semi-structured dance sessions could be mitigated by dance-based exergaming therapy using platforms such as the Kinect (Microsoft Inc., Redmond, WA, USA).

Phase 1 pilots of dance training via exergaming conducted in a laboratory setting have shown improvements in balance control assessed by the Limits of Stability test (NeuroCom International Inc., Clackaman, OR, USA), as well as improvements in other functional clinical measures for individuals with chronic stroke ${ }^{4)}$ and Parkinson's disease ${ }^{3,21)}$. This contemporary dance research has involved the use of electromyography (EMG), force plates, motion analysis using photography, cinematography or videography, and physics analysis ${ }^{2,5}$. However, although these studies, and others, suggest and infer that combining exergaming technology with dance can improve one's volitional CoM and stepping control, they do not directly use a quantitative assessment of kinematic parameters to examine improvements in movement control during dance training itself. Moreover, no study to date has used a continuous motion capture system during dance movement to provide evidence of how different song paces used in dance therapy (or dance-based exergaming) impact full-body movement kinematics. By examining the different biomechanical changes and movement kinematics seen when dancing to different songs, researchers might enhance understanding of motor control and learning during dance based-exergaming, which could enhance the effectiveness and subsequent prescription of dance-training protocols. For example, evaluating dance performance using postural sway and joint range of motion (ROM) could provide critical information to clinicians because these important movement control variables are norm-referenced measures of postural stability and mobility, respectively. Further, it may provide important insights into the qualitative and quantitative ability of the participant to actively imitate and mimic the visually presented dance sequences.

Thus, the purpose of the present study was to establish a quantitative kinematic assessment method for examining postural control and joint kinematics using a commercially available dance-based exergaming Microsoft Kinect 'Just Dance' platform. Specifically, we sought to examine and establish normative values for postural stability (CoM sway area and excursions in both AP and ML directions) and physical mobility (joint angles and excursions in the sagittal plane) during healthy young adults' performance of dance to selected segments of slow-paced (SP), medium-paced (MP), and fast-paced (FP) songs. Since there has not yet been a study of this kind, the young population was chosen to test the feasibility of this paradigm, as well as to serve as a baseline assessment for examining future intervention-related improvements, in order to subsequently replicate a similar protocol in other populations, including aging individuals and those with neurological disorders. The generation of objective baseline data, as opposed to the use of subjective outcome measures, will provide information that could assist in the objective assessment of dance-based exergaming for rehabilitation.

\section{PARTICIPANTS AND METHODS}

Fifteen healthy young adults who were nonprofessional dancers and had no prior involvement in any choreographed dance program as a primary physical activity for at least 1 year (via prescreening interview) provided consent to participate (Table 1). A prior sample size analysis using the $\mathrm{G}^{*}$ Power software package ${ }^{22}$ ) to compare means of anteroposterior CoM and knee joint excursions across the three paces of songs (SP, MP, and FP) was performed using a sub-sample sample size of

Table 1. Demographic data of participants

\begin{tabular}{lcccc}
\hline $\begin{array}{l}\text { Participants } \\
(\mathrm{N}=15)\end{array}$ & $\begin{array}{c}\text { Gender } \\
(\mathrm{M} / \mathrm{F})\end{array}$ & $\begin{array}{c}\text { Age } \\
(\text { years })\end{array}$ & $\begin{array}{c}\text { Weight } \\
(\mathrm{kg})\end{array}$ & $\begin{array}{c}\text { Height } \\
(\mathrm{cm})\end{array}$ \\
\hline Mean & $5 / 10$ & 26.1 & 63.4 & 166.1 \\
$\mathrm{SD}$ & & 4.0 & 8.3 & 8.3 \\
\hline
\end{tabular}


$\mathrm{n}=3$. Based on the mean difference, the resulting effect sizes were 0.75 and 0.77 , respectively, for the anteroposterior CoM excursion and the knee joint excursion, yielding a sample size of $n=13$ to achieve a power of 0.80 . All participants were recruited by posting flyers across various notice boards on the University of Illinois Chicago (UIC) campus. The study was approved by the Institutional Review Board (IRB) and was conducted in the Cognitive, Motor, and Balance Rehabilitation Laboratory (CogMoBal) at UIC.

Participants completed a general health questionnaire to evaluate their health status and family history. Participants were excluded from the study if they reported any recent surgery (less than six months ago) or any musculoskeletal or cardiovascular conditions which may hinder their performance of dance movements.

Participants were exposed to dance movements with segments from Microsoft Kinect 'Just Dance 3' (Microsoft Inc., Redmond, WA, USA). Three songs of slow, medium, and fast pace from the hip-hop genre were selected for testing in this study (Fig. 1). To control for variability of choreography, 30-second segments from each song with similar dance movement sequences were used. The segments were designed to repeat a pre-selected musical passage (a short section of a musical composition), which was synchronized to a specific set of dance movements looped to repeat with the musical passage to ensure reduced variability in the choreography. In general, all three songs' dance movements included forward, backward, and lateral stepping, and the typical dance movements were comprised of rhythmic forward and lateral single- and doublestep touches, sidekicks and steps in-place, hip-hop bounces to front and back, along with in-place and forward marching, necessitating the use of upper and lower body movements in the anteroposterior and mediolateral directions. These dance movements incorporated flexion and extension of the hip, knee and ankle joints, along with flexion and abduction of the shoulder joints. The only difference was the pace of the song and the number of repetitions of a movement sequence. Specifically, the three-song paces were slow-paced (SP-“Dynamite"), medium-paced (MP- "Party Rock Anthem"), and fast-paced (FP- "Price Tag"). Songs from the "hip-hop" genre were chosen due to the genre's wide availability, popularity, and enjoyment in both the United States and worldwide. Song pace was classified as slow (120 bpm), medium (130 bpm), or fast (138 $\mathrm{bpm}$ ) by verifying beats per minute (bpm) for each song using the BPM database ${ }^{23)}$. To learn the dance steps, all participants were initially exposed to two familiarization trials for the dance sequence of each of the three songs before the beginning of the test. The songs were randomly provided to participants to elicit their natural response and performance of the dance steps. The one-time dance session lasted approximately 60 minutes.

All participants had 29 reflective markers (Helen Hayes marker set) placed on specific joints of upper and lower limb segments of their bodies. Dancing to each song was tested and recorded for 30 seconds using an eight-camera motion capture system at 120 frames per second with Cortex-64 3.6.1 software (Motion Analysis, Santa Rosa, CA, USA). The software provides full body kinematic values and computes raw CoM positions and joint angles for each of the participants. To record the number of dance steps for each song, a cost-effective and highly reliable tractivity motion sensor ${ }^{24)}$ (Kineteks Corporation, Vancouver, Canada) was placed on the participants' ankles. Initial processing and analysis of data were performed using an in-house MATLAB code (Math Works, Natick, MA, USA).

We assessed postural stability by evaluating the absolute postural sway area, CoM excursions, number of CoM peaks, and the magnitude of the maximum and minimum CoM peaks in the AP and ML directions. To determine the CoM excursion in both the AP and ML directions, one CoM peak was defined by a complete cycle of upward and downward CoM displacement. The magnitude of the maximum and minimum peaks was measured as the highest and lowest peak heights of the CoM movement, respectively, during a dance performance. Total sway area was defined by the combined AP and ML ellipse area, and the total CoM excursion was defined by the displacement of the CoM in the transverse plane. The primary outcome measures of sway area and excursion were computed using segmental method formulas (shown below) which were proposed by Prieto and colleagues ${ }^{25)}$.

$$
\begin{aligned}
& \text { Total Sway Area }-\mathrm{CE}=\pi \mathrm{ab}=2 \pi \mathrm{F}_{0.05[2, \mathrm{~N}-2]}\left[\mathrm{s}^{2} \mathrm{CoM}_{\mathrm{AP}} \mathrm{s}^{2} \mathrm{CoM}_{\mathrm{ML}}-\mathrm{s}^{2} \mathrm{CoM}_{\mathrm{AP}} \mathrm{CoM}_{\mathrm{ML}}\right]^{1 / 2}, \\
& \text { Total CoM Excursion }=\sum_{n=1}^{N-1}\left(\left(\mathrm{CoM}_{\mathrm{AP}}[\mathrm{n}+1]-\mathrm{CoM}_{\mathrm{AP}}[\mathrm{n}]\right)^{2}+\left(\mathrm{CoM}_{\mathrm{ML}}[\mathrm{n}+1]-\mathrm{CoM}_{\mathrm{ML}}[\mathrm{n}]\right)^{2}\right)^{1 / 2} \\
& \text { where } \mathrm{CE}=95 \% \text { confidence ellipse, } \mathrm{N}=\text { sample size, } \mathrm{CoM}_{\mathrm{AP}}=\mathrm{CoM} \text { in } \mathrm{AP} \text { direction, } \\
& \mathrm{CoM}_{\mathrm{ML}}=\mathrm{CoM} \text { in ML direction, } \mathrm{s}=\text { standard deviation, and } \mathrm{sCoM}_{\mathrm{AP}} \mathrm{CoM}_{\mathrm{ML}}=\text { covariance. }
\end{aligned}
$$

We evaluated physical activity and mobility by determining the absolute number of peak joint angles and the magnitude of the peak joint angles for the hip, knee, and ankle in the sagittal plane. A model created by Vaughan et al. ${ }^{26)}$ was implemented to calculate these joint angles. In this model, the calculation of any joint angle is influenced by defining a reference frame in the proximal and distal segments. For example, the reference point of the hip joint is where the pelvis and thigh meet, the reference point for the knee joint is at the thigh and shank, and the reference point for the ankle joint is at the shank and foot. Joint excursions were then determined by calculating the differences between the maximum and minimum joint angles. The joint angles in the sagittal plane (Fig. 2) were then generated through 3-dimensional trajectories of the segmental markers (from the Helen Hayes Marker set) using a custom-made MATLAB code. After eliminating data from one participant, due to 

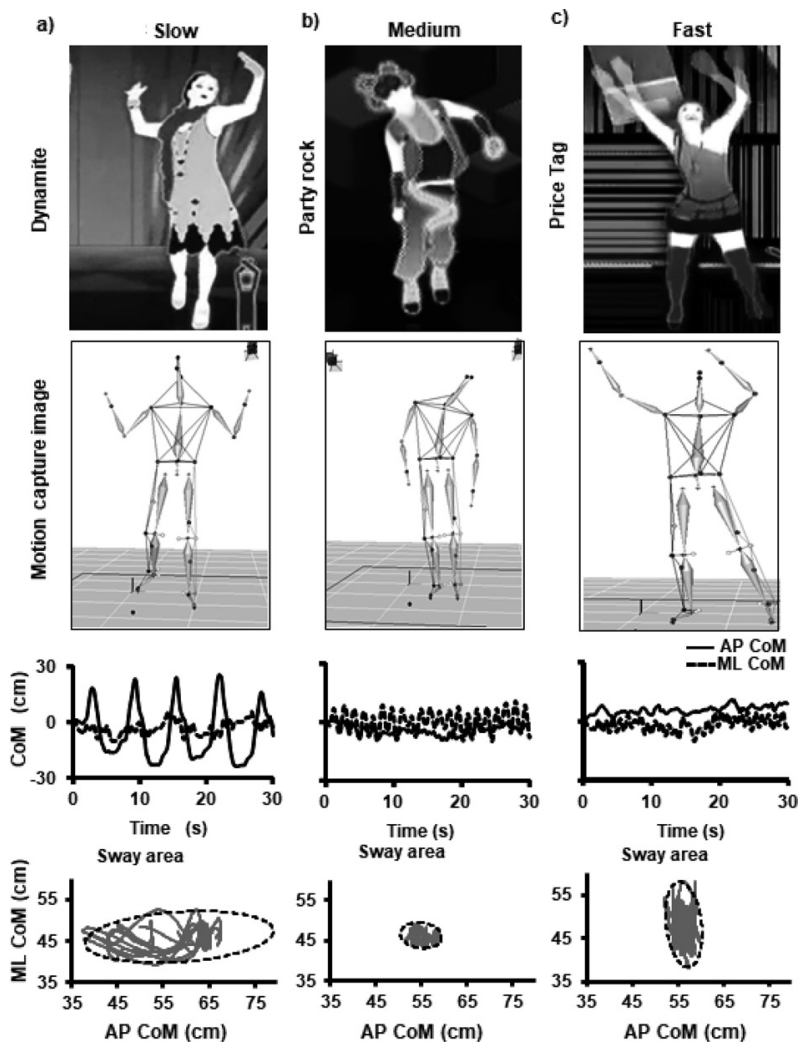
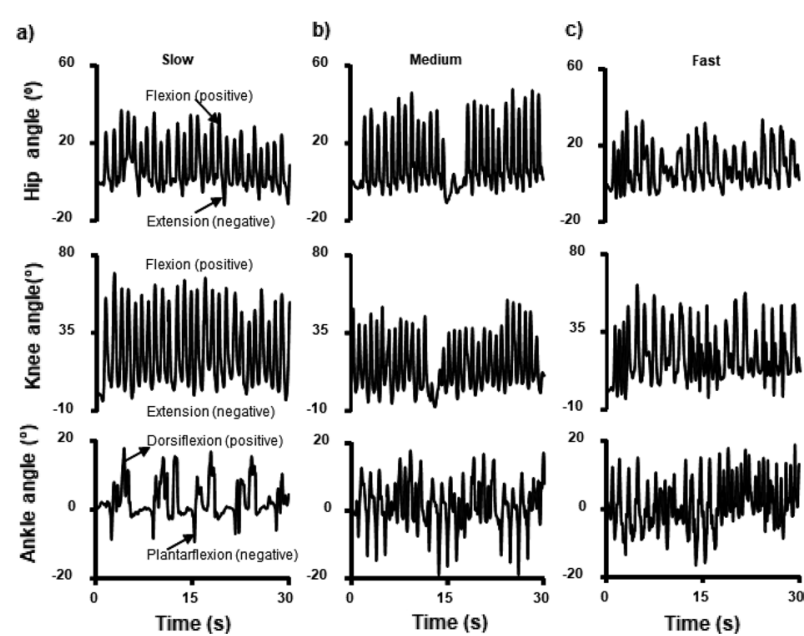

Fig. 2. Representative plots showing hip, knee, and ankle joint excursion angles (in degrees) over time for dance performance to the a) slow-, b) medium-, and c) fast-paced songs. The number of joint peaks increased for dancing to the fast-paced song.

Fig. 1. Representative frontal view of dance video images, motion capture images, anteroposterior (AP) and mediolateral (ML) center of mass (CoM) movement, as well as postural sway area plots for various movements in dance routines from the 'Just Dance' Kinect gaming system used in the study. The CoM plots were normalized to zero in order for the peaks to begin from zero. The figure also shows the titles for the songs used for the a) slow- ("Dynamite"), b) medium- ("Party Rock"), and c) fast-paced ("Price Tag") songs. Songs used for this study were generally from the hip pop genre.

a missing marker data, statistical analysis was conducted using data from the remaining 14 participants.

One-way ANOVA was used to determine variations in total sway area, and total CoM excursions as the song pace changed. Furthermore, a $3 * 2$ repeated measures ANOVA was used to determine the effect of the song on movement in both the AP and ML directions and the interactions between song choice and dance direction, and one-way ANOVA was used to assess the effect of song pace on the CoM peaks and excursions in both the AP and ML directions.

The number of joint angle peaks and joint excursions and the interactions between song choice and extent of joint movement were analyzed with a $3 * 3$ repeated measures ANOVA. Additionally, one-way ANOVA was performed to assess the specific effect of the song pace on the number of peaks and joint excursions for each joint (hip, knee, and ankle). Post hoc analysis was then performed for each song pace to determine variable differences using paired sample t-tests with Bonferroni corrections for controlling multiple comparisons (adjusted significance level of $\alpha=0.02$ ).

Lastly, to determine any significant differences between step counts for each dance, one-way ANOVA was performed. Post hoc analysis was performed using paired t-tests to determine any statistical differences between step count for each of the dance songs (adjusted significance level of $\alpha=0.02$ ).

To ascertain any relationship found between the dance variables, correlation and regression analyses were conducted between variables. Correlations were classified as weak $(\mathrm{R}<0.49)$, moderate $(0.5>\mathrm{R}>0.69)$, or strong $\left.(\mathrm{R}>0.70)^{27}\right)$. With the exception of post hoc tests, all statistical analyses had significance levels set at $\alpha=0.05$, and all statistical analyses were 
performed using Statistical Package for Social Sciences (SPSS) software version 24 (IBM Corporation, Endicott, NY, USA).

\section{RESULTS}

One-way ANOVA showed significant variations in total sway area $(\mathrm{F}(2,41)=4.95, \mathrm{p}<0.01)$ and in total CoM excursions $(\mathrm{F}(2,41)=5.98, \mathrm{p}<0.01)$. For post hoc comparison of total sway area, there was a significantly increased sway area for SP dance compared with both MP dance $(\mathrm{p}<0.04)$ and FP dance $(\mathrm{p}<0.04)$. (Fig. 1 \& Table 2$)$. Post hoc analysis also showed significantly decreased CoM excursions for MP dance compared with both SP and FP dance ( $\mathrm{p}<0.01$ for both) (Table 2).

A $3 * 2$ repeated measures ANOVA showed a significant main effect of song pace on CoM excursion $(\mathrm{F}(2,39)=12.34$, $\mathrm{p}<0.001)$ as well as a significant interaction between song pace and direction of CoM movement $(\mathrm{F}(2,39)=55.25, \mathrm{p}<0.001)$. One-way ANOVA showed a significant effect of song pace on CoM excursions in both the AP $(F(2,41)=18.55$, p $<0.001)$ and ML $(\mathrm{F}(2,41)=55.25, \mathrm{p}<0.001)$ directions. The CoM excursions in both directions for SP dance were significantly greater compared with both MP and FP dance $(\mathrm{p}<0.001)$. Also, CoM excursion was significantly greater in the AP direction than in the ML direction for SP dance $(\mathrm{p}<0.001)$ but was significantly greater in the ML direction than in the AP direction for FP dance $(\mathrm{p}<0.001)$ (Table 2).

A $3 * 2$ repeated measures ANOVA showed a significant main effect of song pace on the number (\#) of CoM peaks (F (2, $39)=5.29, \mathrm{p}<0.01)$. One-way ANOVA showed significant differences in the number of CoM peaks in the ML direction for different song paces $(\mathrm{F}(2,41)=14.61, \mathrm{p}<0.001)$. There was a significantly greater number of peaks in the ML direction for MP dance than for SP dance $(\mathrm{p}<0.001)$, as well as significantly more peaks in the ML direction for FP dance than for SP dance $(\mathrm{p}<0.001)$. However, there were no significant differences in the number of CoM peaks in the AP direction for any of the three-song paces. Additionally, there was a significantly greater number of CoM peaks in the ML direction than in the AP direction for each song pace ( $\mathrm{p}<0.001$ for all) (Table 2$)$.

A $3 * 3$ ANOVA showed a significant main effect of song pace on joint excursions $(F(2,78=25.16, p<0.001)$ and a significant interaction between song pace and extent of movement $(\mathrm{F}(4,78)=5.23, \mathrm{p}<0.001)$. One-way ANOVA demonstrated significant variances between song pace for hip $(F(2,41)=3.51, p<0.04)$, knee $(F(2,41)=7.05, p<0.002)$, and ankle $(F(2$, $41)=4.61, \mathrm{p}<0.02)$ joint excursions. Post hoc comparison showed increased hip and knee joint excursions for MP dance compared with SP dance ( $\mathrm{p}<0.02$ for both). There was also a greater ankle joint excursion for FP dance than for SP dance, a greater knee joint excursion for MP dance than for FP dance, and a greater ankle joint excursion for FP dance than for MP dance ( $p<0.02$ for all). Further, comparisons of joint excursions within each song showed that for SP and MP dance there were significantly increased joint excursions for the knee than for the hip, and these were both greater than the ankle joint excursions $(\mathrm{p}<0.01)$. For FP dance, joint excursion was only significantly greater for the knee than for the ankle $(p<0.01)$ (Table 3).

Results of $3 * 3$ repeated measures ANOVA showed a significant effect of song pace on the number (\#) of joint angle peaks $(\mathrm{F}(2,78)=25.97, \mathrm{p}<0.001)$ and a significant interaction with song $(\mathrm{F}(2,78)=7.64, \mathrm{p}<0.001)$. One-way ANOVA showed significant differences in the number of peaks and excursions of all joints $(\mathrm{p}<0.05$ for all). Post hoc analysis showed a significantly greater number of hip joint peaks for MP dance than for SP dance $(p<0.001)$. The number of knee joint peaks was significantly greater for FP dance than for MP and SP dance ( $p<0.001$ for both). Additionally, the number of ankle joint peaks was significantly greater for FP dance than for SP dance $(\mathrm{p}<0.001)$. There were significantly greater numbers of ankle joint peaks than hip joint peaks for both MP and FP dance $(\mathrm{p}<0.003$ for both). Also, for FP dance, the number of peaks was significantly greater for the knee than for the hip $(\mathrm{p}<0.001)$ (Table 3$)$.

One-way ANOVA demonstrated a significant effect of song pace on the number of dance steps taken $(\mathrm{F}(2,41)=31.69$, $\mathrm{p}<0.001)$. The number of dance steps was also seen to be strongly correlated with song pace $(\mathrm{F}(2,41)=18.68, \mathrm{R}=0.72$, $\mathrm{p}<0.001)$. The number of dance steps increased from SP dance $(\mathrm{M}=33.26, \mathrm{SD}=7.19)$ to $\mathrm{MP}$ dance $(\mathrm{M}=41.33, \mathrm{SD}=4.40)$ to $\mathrm{FP}$ dance $(M=47.92, S D=6.31)$, and this increasing pattern was signficant $(\mathrm{p}<0.003)$.

Linear regression analysis showed a non-significant, weak negative correlation between the CoM excursions in the AP and ML directions $(\mathrm{F}(1,26)=2.92, \mathrm{R}=-0.30, \mathrm{p}>0.05)$ (Table 4). Another linear regression analysis showed a significant, moderate negative relationship between CoM excursions and the number of CoM peaks in the AP direction $(\mathrm{F}(1,26)=19.23, \mathrm{R}=$ $-0.65, \mathrm{p}<0.05$ ) (Table 4). Simultaneously, the results showed that the number of AP CoM peaks had a significant, moderate positive correlation with the number of dance steps $(\mathrm{F}(1,26)=14.64, \mathrm{R}=0.61, \mathrm{p}<0.05)$. In addition, the results revealed that the number of ML CoM peaks had some weak positive correlative trend with step count, but this was not significant (F (1, 26) $=2.34, \mathrm{R}=0.09, \mathrm{p}>0.05$ ) (Table 5). Correlation analysis demonstrated a significant, weak positive association between the number of dance steps and the number of hip angle peaks $(\mathrm{F}(1,26)=4.41, \mathrm{R}=0.39, \mathrm{p}<0.05)$, as well as a nonsignificant weak positive association with hip excursion $(\mathrm{F}(1,26)=0.012, \mathrm{R}=0.03, \mathrm{p}>0.05)$. Further analysis revealed that the number of dance steps was significantly, moderately positively correlated with the number of knee angle peaks $(\mathrm{F}(1,26)=23.18, \mathrm{R}=0.69$, $\mathrm{p}<0.05)$, but that there was no significant association with knee excursion $(\mathrm{F}(1,26)=0.46, \mathrm{R}=0.14, \mathrm{p}>0.05)$. Lastly, results demonstrated a significant, moderate positive correlation between the number of dance steps and the number of ankle peaks $(\mathrm{F}(1,26)=9.44 ; \mathrm{R}=0.52, \mathrm{p}<0.05)$, and a similar trend was also seen when comparing the number of steps to ankle excursions $(\mathrm{F}(1,26)=18.68 ; \mathrm{R}=0.66, \mathrm{p}<0.05)$ (Tables 4 and 5). 
Table 2. Postural stability measures of the dance-based exergaming

\begin{tabular}{lccc}
\hline Postural stability variables & SP & MP & FP \\
\hline Total Sway Area $\left(\mathrm{cm}^{2}\right)$ & $1,077.6 \pm 209.9^{*}$ & $212.9 \pm 346.0$ & $314.1 \pm 133.6^{*}$ \\
Total CoM excursion $(\mathrm{cm})$ & $629.8 \pm 380.5^{*}$ & $311.2 \pm 119.5$ & $478.5 \pm 149.0^{*}$ \\
AP CoM excursion $(\mathrm{cm})$ & $45.7 \pm 18.2^{*+}$ & $10.6 \pm 4.5$ & $12.9 \pm 4.1$ \\
ML CoM excursion $(\mathrm{cm})$ & $15.0 \pm 6.5$ & $12.6 \pm 2.9$ & $23.0 \pm 5.6^{*}$ \\
\# AP CoM peaks & $5.6 \pm 1.3$ & $7.3 \pm 1.1$ & $8.8 \pm 1.5$ \\
\# ML CoM peaks & $19.0 \pm 4.1^{+}$ & $23.9 \pm 3.5^{*+}$ & $20.3 \pm 3.5^{+}$ \\
\hline
\end{tabular}

Values are (Mean $\pm \mathrm{SD}$ ) for variables, $\mathrm{p}<0.05$. SP: Slow-paced song; MP: Medium-paced song; FP: Fast-paced song; AP: anteroposterior; ML: mediolateral; CoM: center of mass. * denotes significantly greater values within the same variable among the three song paces, + denotes significantly greater values between different variables within a song pace only, and ${ }^{*+}$ denotes significantly greater values within the same variable among songs and between variables within a song.

Table 3. Joint excursion, peaks, and step count during dance-based exergaming

\begin{tabular}{lccc}
\hline Mobility and physical activity variables & SP & MP & FP \\
\hline Joint excursion (degrees) & & & \\
Hip & $40.6 \pm 11.0^{*}$ & $50.4 \pm 8.5^{\mathrm{a}, \mathrm{c}}$ & $44.2 \pm 10.1$ \\
Knee & $55.5 \pm 15.5^{+,-}$ & $83.1 \pm 14.9^{\mathrm{a}, \mathrm{c},+,}$ & $60.2 \pm 23.2^{+,-}$ \\
Ankle & $32.2 \pm 12.7$ & $34.3 \pm 21.3$ & $46.7 \pm 21.7^{\mathrm{b}, \mathrm{c}}$ \\
\# Joint peaks & & & \\
Hip & $15.7 \pm 1.7$ & $21.1 \pm 3.8^{\mathrm{a}}$ & $18.3 \pm 1.5$ \\
Knee & $20.6 \pm 3.5$ & $25.6 \pm 4.8^{\mathrm{a}}$ & $30.8 \pm 6.3^{\mathrm{b}, \mathrm{c},-}$ \\
Ankle & $25.7 \pm 7.5$ & $33.2 \pm 9.5^{\mathrm{a},{ }^{*}}$ & $33.1 \pm 6.7^{\mathrm{b}^{*}}$ \\
\# Steps $\left(\mathrm{R}_{\text {pace }}=0.72, \mathrm{p}<0.05\right)$ & $33.3 \pm 5.1$ & $41.3 \pm 7.3^{\mathrm{i}}$ & $47.9 \pm 4.8^{\mathrm{ii}, \mathrm{iii}}$ \\
\hline
\end{tabular}

Values are (Mean $\pm \mathrm{SD}$ ) of variables, $\mathrm{p}<0.05$. SP: Slow-paced song; MP: Medium-paced song; FP: Fast-paced song. Significantly greater values are shown by; 1) Between songs in each joint: ${ }^{\text {aSP }}$ vs. MP, ${ }^{\mathbf{b}} \mathrm{SP}$ vs. FP, ${ }^{\mathbf{c}} \mathrm{MP}$ vs. FP, 2) Between the

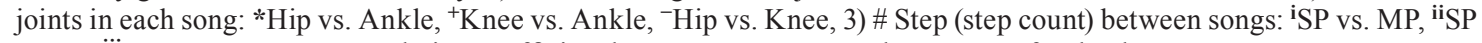
vs. FP, ${ }^{i i i} M P$ vs. FP. $R_{\text {pace }}=$ correlation coefficient between song pace and step count for the dance.

Table 4. Correlations ( $\mathrm{R})$ between postural variables

\begin{tabular}{lccccc}
\hline & $\begin{array}{c}\text { AP CoM } \\
\text { excursion }\end{array}$ & $\begin{array}{c}\text { ML CoM } \\
\text { excursion }\end{array}$ & $\begin{array}{c}\text { \# AP CoM } \\
\text { peaks }\end{array}$ & $\begin{array}{c}\text { \# ML CoM } \\
\text { peaks }\end{array}$ & \# Steps \\
\hline AP CoM excursion & & -0.30 & $-0.65^{*}$ & & \\
ML CoM excursion & -0.30 & & & 0.10 & $0.61^{*}$ \\
\# AP CoM peaks & $-0.65^{*}$ & & & & 0.30 \\
\# ML CoM peaks & & -0.10 & & 0.30 & \\
\# Steps & & & $0.61^{*}$ & 0.30 \\
\hline
\end{tabular}

AP: anteroposterior; ML: mediolateral; CoM: center of mass.

Values are correlation coefficients $(\mathrm{R})$ between variables, ${ }^{*}$ denotes $\mathrm{p}<0.05$.

Table 5. Correlations (R) between joint mobility variables

\begin{tabular}{ll}
\hline & \# Steps \\
\hline Hip excursion & 0.30 \\
Knee excursion & 0.14 \\
Ankle excursion & $0.66^{*}$ \\
\# Hip peaks & $0.39^{*}$ \\
\# Knee peaks & $0.69^{*}$ \\
\# Ankle peaks & $0.52^{*}$ \\
\hline
\end{tabular}

Values are correlation coefficients $(\mathrm{R})$ between variables,

$*$ denotes $\mathrm{p}<0.05$. 


\section{DISCUSSION}

The present study evaluated determinants of postural stability and physical mobility (postural sway, CoM excursion, and joint angle changes) to establish a quantitative assessment method with dance-based exergaming using three different paces/ intensities of dance movements (SP, MP, and FP) in healthy young adults. The study was able to generate normative values of postural stability (CoM sway area and excursions in both AP and ML directions) and physical mobility (joint angles and excursions in the sagittal plane) during healthy young adults' performance of dance to selected segments of slow-paced (SP), medium-paced (MP), and fast-paced (FP) songs.

When dancing, individuals must shift their CoM to different spatial locations, change their movement speed, and synchronize their multi-limb segments with audiovisual stimuli, all while simultaneously controlling their balance. Because SP dance had the least bpm, participants were able to maintain the pace and rhythm of the SP song while simultaneously attaining a greater sway area and AP CoM excursion than for the other two song paces. The increased number of beats per minute with MP and FP provided some difficulty for participants to rapidly shift their CoM to its maximum level due to the limited time for movement transitions, resulting in reduced sway area and excursions (Fig. 1 \& Table 2). However, the FP dance did show comparatively higher ML CoM excursion (Table 2), but this could merely indicate that the FP dance provided via the Kinect system required the participants to accomplish increased excursions in the ML direction to complete the dance steps. Likewise, the equivalent AP and ML CoM excursions for the MP song could imply that the MP dance, "Party Rock," potentially required the execution of balanced movements in both the AP and ML directions (Fig. 1a). Given that the capacity to maintain balance control during functional tasks is acquired through AP and ML stability ${ }^{28)}$, a comprehensive dance-based exergaming assessment protocol enlisting both SP and FP dancing could enhance the testing of dynamic balance control in the AP and ML directions, respectively.

While comparatively smaller corresponding CoM excursions accompanied a higher number of CoM peaks in the AP direction for all three dance paces, no such association was observed in the ML direction (Table 4). This could be because all three songs were potentially able to facilitate similar trends for CoM excursions and peaks in the ML direction. Previous studies have indicated that ML CoM motion is associated with maintaining dynamic stability and is a functional indicator of balance maintenance during motor tasks ${ }^{29}, 30$ ). With this in mind, it is possible that the young adults in this study exhibited a compensatory adjustment strategy to establish dynamic stability while dancing which caused a similar number of CoM peaks and similar CoM excursions in the ML direction. Ojofeitimi et al. ${ }^{31)}$ demonstrated that skilled dancers had significantly increased ML CoM displacement during a weight shifting task, exhibiting higher mobility control ability than amateur dancers. It is also known that ML postural control is essential for rapid voluntary weight shifting to different spatial locations while maintaining one's balance ${ }^{32}$, and, therefore, it could be postulated that all three song intensities would be useful for assessment of ML postural control.

Additionally, the number of joint peaks and joint excursions are useful for evaluating joint mobility. In particular, joint mobility provides information on the capacity to adhere to and perform dance movement sequences with respect to the song pace. The joint excursion pattern seen for all three dance songs was that the highest excursion exhibited was for knee flexion, followed by hip flexion and ankle plantarflexion ${ }^{33}$. Similar to our study results, other dance and gait studies have also found similar patterns of maximum joint excursions ${ }^{33,34)}$. Therefore, it is possible that the songs used in our dance-based exergaming may play an important role in enhancing some key determinants of gait. As joint excursion indicates the total size of the free joint angle needed to perform tasks, the results (greatest excursion of knee joint with MP) may indicate that MP may be suitable for rehabilitation of knee movement in gait training and also in performing functional activities such as sit-to-stand tasks and stair climbing ascent and descent. Also, because the number of joint peaks represent the frequency of the joints' ability to reach maximum joint angles, the results (increased number of joint peaks for the knee and ankle with MP and FP) may imply that MP and FP could be applicable for training gait speed or other activities which require speed for proper execution.

Furthermore, the results demonstrated a positive correlation between the number of AP CoM peaks and the number of dance steps (Table 4). Improvements in the AP direction may play a critical role in augmenting balance and propulsion for improved gait, and, because this study evaluated the number of steps as a measure of physical activity, physical activity was also seen to increase with these AP improvements. Also, increases in step count could play a critical role in improving cardiovascular endurance, which is important for reducing inactivity and comorbidities in the general population ${ }^{28,35)}$. Regular physical activity is known to increase the level of fitness in healthy and older population ${ }^{36,37)}$, and dance-based exergaming could also play a significant role in this aspect. Thus, the use of a commercially available, off-the-shelf, and cost-effective Kinect gaming system for dance-based exergaming as an assessment tool for physical activity and cardiovascular endurance could offer immense possibilities for both physical activity and rehabilitation. Albeit, this assertion may need further studies to authenticate this claim.

This study demonstrated that the dance assessment protocol resulted in increased dance steps as song pace increased and that the number of dance steps was positively correlated with the number of knee and ankle joint angle peaks as well as with ankle excursion (Table 5). This increase in the number of knee and ankle joint angle peaks, along with a higher ankle joint excursion, could suggest that such assessment protocol might be beneficial for testing changes most likely at the knee and 
ankle level but less likely at the hip joint (Table 5). However, studies have also indicated that increases in knee and ankle range of motion are correlated with increased gait velocities in both young and older adults ${ }^{29,34)}$, suggesting that dance-based exergaming assessment could potentially play a role in testing gait speed, but this needs further investigation. Also, this study was able to generate normative data for CoM excursions and joint excursions for different intensities of dance songs used for dance-based exergaming assessment. Dance therapy has been very effective for neurological conditions, and, because of this, kinematic evaluation of dance steps could be a sensitive or objective quantification of pre-post training effect. Successful walking ability primarily involves a substantial single support period, and so control of ML balance is essential, particularly when transitioning support from one limb to the other ${ }^{38)}$. Secondly, when walking, the ability to regain equilibrium under more dynamic conditions, such as upon an unexpected perturbation of the body's position (e.g., a trip or slip), is vital for preventing falls. This dance assessment protocol with our chosen song paces would likely offer a sensitive and objective method of evaluating postural stability and risk of falling before and after dance training sessions. However, further research is needed to ascertain the above-stated postulations.

The results of this study may be limited by a few factors, and therefore the findings should be interpreted with caution. One notable limitation was the relatively small sample size of the study. However, the effect sizes for CoM excursion and knee joint excursion for the comparisons performed were 0.78 and 0.76 and yielded a power of 0.88 and 0.87 with the current sample size. These large effect sizes obtained in the study are in line with the clinical study guide to statistics which states that an effect size greater than 0.70 is appropriate for obtaining normative values ${ }^{39}$. Also, the current study involved three songs of different paces from the hip-hop genre using the Kinect exergaming system, thus the results do not reflect dance kinematics across all dance genres and cannot be generalized. Future studies should conduct a similar kinematic analysis of dance with other genres. Additionally, the present study was conducted on healthy young adults', and prospective studies should also include aging and neurologically-impaired populations to understand their ability to reproduce dance movements with the effect of aging and neurological deficits.

In recent times, exergaming-based dance interventions with different genres (styles) and paces (intensities) have gained momentum in their applications for rehabilitation among older adults and individuals with neurological conditions. Advancing knowledge in such areas as the biomechanics of dance would be an addition to dance therapy literature by ensuring that interventions provide appropriate execution of dance movements so that individuals gain optimal efficiency while training. This study was able to establish movement kinematics of postural control and joint mobility using three songs of different paces provided via dance-based exergaming among healthy young adults. These findings are beneficial in that they provide clinicians with insight into dance movement strategies using the Kinect exergaming system.

\section{Funding}

There was no funding for this study.

\section{Conflict of interest}

The authors declare that there is no conflict of interest.

\section{ACKNOWLEDGMENTS}

The authors thank the Research Open Access Publishing (ROAAP) Fund of the University of Illinois at Chicago for the financial support towards the open access publishing fee for this article. Thank you to all of the researchers and students at the Cognitive, Motor, and Balance Rehabilitation Laboratory (CogMoBal) for their support during the data collection and analysis. We are also extremely grateful to Alison Schenone for her tremendous work in editing the final draft of this manuscript. Special thanks to Dr. Tanvi Bhatt for her assistance and advice.

\section{REFERENCES}

1) Krampe J: Exploring the effects of dance-based therapy on balance and mobility in older adults. West J Nurs Res, 2013, 35: 39-56. [Medline] [CrossRef]

2) Hackney ME, Earhart GM: Effects of dance on movement control in Parkinson's disease: a comparison of Argentine tango and American ballroom. J Rehabil Med, 2009, 41: 475-481. [Medline] [CrossRef]

3) Lee NY, Lee DK, Song HS: Effect of virtual reality dance exercise on the balance, activities of daily living, and depressive disorder status of Parkinson's disease patients. J Phys Ther Sci, 2015, 27: 145-147. [Medline] [CrossRef]

4) Subramaniam $\mathrm{S}$, Bhatt T: Does a virtual reality-based dance training paradigm increase balance control in chronic stroke survivors? A preliminary study. Int J Neurorehabil, 2015, 2: 1-10. [CrossRef]

5) Pichierri G, Murer K, de Bruin ED: A cognitive-motor intervention using a dance video game to enhance foot placement accuracy and gait under dual task conditions in older adults: a randomized controlled trial. BMC Geriatr, 2012, 12: 74. [Medline] [CrossRef]

6) Burkhardt J, Brennan C: The effects of recreational dance interventions on the health and well-being of children and young people: a systematic review. Arts Health, 2012, 4: 148-161. [CrossRef] 
7) Shigematsu R, Chang M, Yabushita N, et al.: Dance-based aerobic exercise may improve indices of falling risk in older women. Age Ageing, 2002, 31: 261-266. [Medline] [CrossRef]

8) Sofianidis G, Hatzitaki V, Douka S, et al.: Effect of a 10-week traditional dance program on static and dynamic balance control in elderly adults. J Aging Phys Act, 2009, 17: 167-180. [Medline] [CrossRef]

9) Keogh JW, Kilding A, Pidgeon P, et al.: Physical benefits of dancing for healthy older adults: a review. J Aging Phys Act, 2009, 17: 479-500. [Medline] [CrossRef]

10) Federici A, Bellagamba S, Rocchi MB: Does dance-based training improve balance in adult and young old subjects? A pilot randomized controlled trial. Aging Clin Exp Res, 2005, 17: 385-389. [Medline] [CrossRef]

11) Veronese N, Maggi S, Schofield P, et al.: Dance movement therapy and falls prevention. Maturitas, 2017, 102: 1-5. [Medline] [CrossRef]

12) da Silva Borges EG, de Souza Vale RG, Cader SA, et al.: Postural balance and falls in elderly nursing home residents enrolled in a ballroom dancing program. Arch Gerontol Geriatr, 2014, 59: 312-316. [Medline] [CrossRef]

13) Betker AL, Desai A, Nett C, et al.: Game-based exercises for dynamic short-sitting balance rehabilitation of people with chronic spinal cord and traumatic brain injuries. Phys Ther, 2007, 87: 1389-1398. [Medline] [CrossRef]

14) Lohse KR, Hilderman CG, Cheung KL, et al.: Virtual reality therapy for adults post-stroke: a systematic review and meta-analysis exploring virtual environments and commercial games in therapy. PLoS One, 2014, 9: e93318. [Medline] [CrossRef]

15) Fernández-Argüelles EL, Rodríguez-Mansilla J, Antunez LE, et al.: Effects of dancing on the risk of falling related factors of healthy older adults: a systematic review. Arch Gerontol Geriatr, 2015, 60: 1-8. [Medline] [CrossRef]

16) Hwang PW, Braun KL: The effectiveness of dance interventions to improve older adults' health: a systematic literature review. Altern Ther Health Med, 2015, 21: 64-70. [Medline]

17) Sharp K, Hewitt J: Dance as an intervention for people with Parkinson's disease: a systematic review and meta-analysis. Neurosci Biobehav Rev, 2014, 47 : 445-456. [Medline] [CrossRef]

18) Granacher U, Muehlbauer T, Bridenbaugh SA, et al.: Effects of a salsa dance training on balance and strength performance in older adults. Gerontology, 2012, 58: 305-312. [Medline] [CrossRef]

19) Harris N, Cronin J, Keogh J: Contraction force specificity and its relationship to functional performance. J Sports Sci, 2007, 25: 201-212. [Medline] [CrossRef]

20) Schoene LM: Biomechanical evaluation of dancers and assessment of their risk of injury. J Am Podiatr Med Assoc, 2007, 97: 75-80. [Medline] [CrossRef]

21) Šumec R, Filip P, Sheardová K, et al.: Psychological benefits of nonpharmacological methods aimed for improving balance in Parkinson's disease: a systematic review. Behav Neurol, 2015, 2015: 620674. [Medline] [CrossRef]

22) Erdfelder E, Faul F, Buchner A: GPOWER: a general power analysis program. Behav Res Methods Instrum Comput, 1996, 28: 1-11. [CrossRef]

23) BPM D: BPM Database. 2003-2017.

24) Warburton D, Jeklin A, Bredin S: The validity of the tractivity motion sensor during walking. Health Fit J Can, 2013,6 : 114-122.

25) Prieto TE, Myklebust JB, Hoffmann RG, et al.: Measures of postural steadiness: differences between healthy young and elderly adults. IEEE Trans Biomed Eng, 1996, 43: 956-966. [Medline] [CrossRef]

26) Vaughan CL, Davis BL, Jeremy C: Dynamics of human gait. 1999.

27) Nangolo C, Musingwini C: Empirical correlation of mineral commodity prices with exchange-traded mining stock prices. J S Afr Inst Min Metall, 2011, 111: 459-468.

28) Latt MD, Menz HB, Fung VS, et al.: Walking speed, cadence and step length are selected to optimize the stability of head and pelvis accelerations. Exp Brain Res, 2008, 184: 201-209. [Medline] [CrossRef]

29) Orendurff MS, Segal AD, Klute GK, et al.: The effect of walking speed on center of mass displacement. J Rehabil Res Dev, 2004, 41: 829-834. [Medline] [CrossRef]

30) Fidler A, Haddad JM, Gagnon JL: Postural control strategies in dancers and non dancers. ISBS-Conference Proceedings Archive, $2008,1$.

31) Ojofeitimi S, Bronner S, Spriggs J, et al.: Effect of training on postural control and center of pressure displacement during weight shift. J Orthop Sports Phys Ther, 2003, 33: 115 .

32) Chou LS, Kaufman KR, Hahn ME, et al.: Medio-lateral motion of the center of mass during obstacle crossing distinguishes elderly individuals with imbalance. Gait Posture, 2003, 18: 125-133. [Medline] [CrossRef]

33) Kerrigan DC, Todd MK, Della Croce U: Gender differences in joint biomechanics during walking: normative study in young adults. Am J Phys Med Rehabil, 1998, 77: 2-7. [Medline] [CrossRef]

34) Bronner S, Ojofeitimi S: Gender and limb differences in healthy elite dancers: passé kinematics. J Mot Behav, 2006, 38: 71-79. [Medline] [CrossRef]

35) Tudor-Locke C, Bassett DR Jr: How many steps/day are enough? Preliminary pedometer indices for public health. Sports Med, 2004, 34: 1-8. [Medline] [CrossRef]

36) Gordon NF, Gulanick M, Costa F, et al. American Heart Association Council on Clinical Cardiology, Subcommittee on Exercise, Cardiac Rehabilitation, and Prevention; the Council on Cardiovascular Nursing; the Council on Nutrition, Physical Activity, and Metabolism; and the Stroke Council: Physical activity and exercise recommendations for stroke survivors: an American Heart Association scientific statement from the Council on Clinical Cardiology, Subcommittee on Exercise, Cardiac Rehabilitation, and Prevention; the Council on Cardiovascular Nursing; the Council on Nutrition, Physical Activity, and Metabolism; and the Stroke Council. Stroke, 2004, 35: 1230-1240. [Medline] [CrossRef]

37) Billinger SA, Arena R, Bernhardt J, et al. American Heart Association Stroke Council, Council on Cardiovascular and Stroke Nursing, Council on Lifestyle and Cardiometabolic Health, Council on Epidemiology and Prevention, Council on Clinical Cardiology: Physical activity and exercise recommendations for stroke survivors: a statement for healthcare professionals from the American Heart Association/American Stroke Association. Stroke, $2014,45: 2532-2553$. [Medline] [CrossRef]

38) Neptune RR, Kautz SA, Zajac FE: Contributions of the individual ankle plantar flexors to support, forward progression and swing initiation during walking. J Biomech, 2001, 34: 1387-1398. [Medline] [CrossRef]

39) Ghaemi SN: A clinician's guide to statistics and epidemiology in mental health: measuring truth and uncertainty. Cambridge University Press, 2009. 